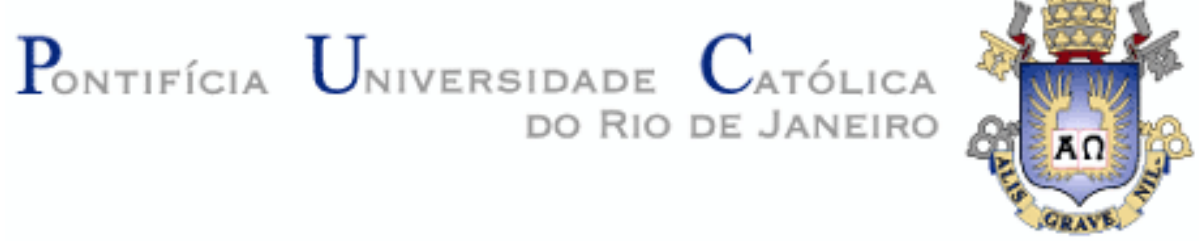

Sara Solange Parga Carneiro

Planejamento do sequenciamento de caminhões em um ambiente de produção sob encomenda

Dissertação de Mestrado

Dissertação apresentada como requisito parcial para obtenção do grau de Mestre pelo Programa de Pós-Graduação em Engenharia de Produção da PUC-Rio.

Orientador: Prof ${ }^{a}$ Fernanda Maria Pereira Raupp

Rio de Janeiro

Fevereiro de 2013 
Sara Solange Parga Carneiro

\section{Planejamento do sequenciamento de caminhões em um ambiente de produção sob encomenda}

Dissertação apresentada como requisito parcial para obtenção do grau de Mestre pelo Programa de Pós-Graduação em Engenharia de Produção da PUC-Rio. Aprovada pela Comissão Examinadora abaixo assinada.

Prof ${ }^{a}$. Fernanda Maria Pereira Raupp

Orientadora

Departamento de Engenharia Industrial - PUC-Rio

Prof?. Márcia Helena Costa Fampa

Departamento de Ciências da Computação - UFRJ

Prof. Eduardo Uchoa Barboza

Escola de Engenharia, Centro Tecnológico - UFF

Prof. José Eugênio Leal

Coordenador Setorial

Departamento de Engenharia Industrial - PUC-Rio

Rio de Janeiro, 04 de fevereiro de 2013 
Todos os direitos reservados. É proibida a reprodução total ou parcial do trabalho sem autorização da universidade, da autora e do orientador.

\section{Sara Solange Parga Carneiro}

Graduou-se em Engenharia de Produção pela Universidade do Estado do Pará (UEPa) no ano de 2010. Atualmente desempenha atividades na área de Compras em uma empresa montadora de caminhões no ABC paulista.

Ficha Catalográfica

\begin{tabular}{|c|}
\hline Carneiro, Sara Solange Parga \\
\hline $\begin{array}{l}\text { Planejamento do sequenciamento de } \\
\text { caminhões em um ambiente de produção sob } \\
\text { encomenda / Sara Solange Parga Carneiro ; } \\
\text { orientador: Fernanda Maria Pereira Raupp. }-2013 . \\
75 \text { f. : il. (color.) ; } 30 \mathrm{~cm}\end{array}$ \\
\hline $\begin{array}{l}\text { Dissertação } \\
\text { Universidade Católica do Restrado)-Pontifícia } \\
\text { Departamento de Engenharia Industrial, } 2013 . \\
\text { Inclui bibliografia }\end{array}$ \\
\hline $\begin{array}{l}\text { 1. Engenharia Industrial }- \text { Teses. } 2 . \\
\text { Sequenciamento. 3. Montadora de caminhão. } 4 . \\
\text { Sequenciamento de carros. 5. Otimização. I. Raupp, } \\
\text { Fernanda Maria Pereira. II. Pontifícia Universidade } \\
\text { Católica do Rio de Janeiro. Departamento de } \\
\text { Engenharia Industrial. III. Título. }\end{array}$ \\
\hline
\end{tabular}

CDD: 658.5 
À minha mãe, pelo seu exemplo. 


\section{Agradecimentos}

Obrigada, Pai pela sabedoria e graças ofertadas.

Devo agradecimentos a todos que diretamente, ou mesmo indiretamente, contribuíram para a realização deste trabalho.

Obrigada, em especial: à minha orientadora, Fernanda Raupp - pela paciência, insistência e conselhos; à minha irmã, Savana Carneiro - que soube dividir seu conhecimento; minha mãe, Graça Parga - pelo zelo, pelo apoio incondicional e pelo exemplo de uma vida inteira; meu namorado, Salim Rabelo - principal ouvinte e parceiro de proveitosas discussões e, ainda, aos amigos - pelos incontáveis incentivos.

Não poderia deixar de mencionar que este trabalho não seria realizado sem o apoio do Conselho Nacional de Desenvolvimento Científico e Tecnológico $\mathrm{CNPq}$ - Brasil, através da bolsa de estudos concedida. 


\section{Resumo}

Carneiro, Sara Solange Parga; Raupp, Fernanda Maria Pereira. Planejamento do sequenciamento de caminhões em um ambiente de produção sob encomenda. Rio de Janeiro, 2013. 75p. Dissertação de Mestrado - Departamento de Engenharia Industrial, Pontifícia Universidade Católica do Rio de Janeiro.

A maioria das pesquisas científicas publicadas sobre sequenciamento da produção na indústria automobilística consideram os pedidos já alocados em um dia ou turno de trabalho, desconsiderando especificidades do planejamento da cadeia de suprimentos. Esta dissertação procura contribuir no campo do planejamento da produção, propondo um modelo matemático de programação inteira mista que aborda de maneira integrada dois problemas de otimização fundamentais da cadeia: o problema de seleção de pedidos e o problema de sequenciamento de carros em uma única linha de montagem. A fim de abordar questões bem próximas a realidade, incluindo apresentação de experimentos numéricos com o modelo proposto, utilizou-se como cenário o segmento de caminhões, dentro da indústria automotiva. Considerou-se, como objetivo no modelo, além das abordagens tradicionais (minimizar sobrecarga de trabalho, troca de cores e violação de restrições), a demanda dos clientes com relação a prazos de entrega do pedido - principal reforço para uma indústria que pretende cada vez mais migrar para um ambiente de produção orientado pela demanda.

\section{Palavras-chave}

sequenciamento; montadora de caminhão; sequenciamento de carros; otimização. 


\section{Abstract}

Carneiro, Sara Solange Parga; Raupp, Fernanda Maria Pereira (Advisor). Planning of truck sequencing in assembly-to-order production environment. Rio de Janeiro, 2013. 75p. MSc. Dissertation - Departamento de Engenharia Industrial, Pontifícia Universidade Católica do Rio de Janeiro.

Most published scientific research on production sequencing in the automotive industry consider orders already allocated in a day or shift, disregarding specificities of supply chain planning. This paper aims to contribute in production planning field, proposing a mathematical model of mixed-integer programming that addresses in a integrated way two fundamental problems from chain: the problem of order selection and car sequencing problem on a single assembly line. In order to approach practical issues, including presentation of numerical experiments from proposed model, the truck segment within the automotive industry was used as scenario. It was considered as objective in the model, beyond traditional approaches (minimize work overload, color changing and violation of restrictions), customer demand with respect promised due dates, the main reinforcement for an industry that increasingly want migrate to a production environment driven by demand.

\section{Keywords}

sequencing; truck automaker; car sequencing problem; optimization. 



\section{Sumário}

1 Introdução 13

$\begin{array}{ll}\text { 1.1. Metodologia } & 14\end{array}$

1.2. Objetivo Geral 14

1.2.1. Objetivos específicos 15

$\begin{array}{ll}\text { 1.3. Estrutura da dissertação } & 15\end{array}$

2 Revisão da Literatura 16

2.1. Gerenciamento da cadeia de suprimentos automotiva 18

2.1.1. Particularidades da demanda nas montadoras de caminhões 22

2.2. Sequenciamento em linhas de montagem de modelos mistos 25

2.2.1. O problema de sequenciamento de carros 29

3 Modelo matemático 35

3.1. Sobre o balanceamento da linha de montagem 36

3.1.1. Formalização do modelo 39

3.2. Sobre as limitações físicas da linha de montagem 40

3.2.1. Formalização do modelo 42

3.3. Sobre o agrupamento de cores 43

3.3.1. Formalização do modelo 45

3.4. Sobre o espaçamento de opcionais 45

3.4.1. Formalização do modelo 46

3.5. Experimento Computacional - Primeira parte 47

3.6. Sobre definições de datas limites para entrada na produção 49

3.6.1. Formalização do modelo 50

3.7. Sobre a definição de data de produção para uma configuração específica $\quad 51$

3.7.1. Formalização do modelo 52

3.8. Experimento Computacional - Segunda Parte 52

3.9. Sobre nível de demanda além da capacidade de produção 54

3.9.1. Formulação do modelo 55

3.10. Sobre nível de demanda aquém da capacidade 56 
3.10.1. Formulação do modelo

3.11. Experimento Computacional - Terceira Parte 58

3.12.

60

Adição de peso nas parcelas da função objetivo

60

4 Conclusão

62

4.1. Sugestão para trabalhos futuros

63

5 Referências Bibliográficas

65

6 Apêndice

68 


\section{Lista de Figuras}

Figura 1 - Representação de uma linha de montagem contínua

Figura 2 - Atividades e prazos de planejamento da indústria automotiva 18 Figura 3 - Simulação do Planejamento Mestre da Produção 20

Figura 4 - Gráfico da evolução da produção e licenciamento de caminhões no Brasil

Figura 5 - Gráfico da variação da produção de caminhões no Brasil e PIB

Figura 6 - Gráfico da evolução de publicações acadêmicas sobre o PSC

Figura 7 - Representação da ocorrência de opcionais na linha de produção

Figura 8 - Representação da contabilização de violação do opcional " + " 31

Figura 9 - Cenário 1 sobre o balanceamento da linha de produção

Figura 10 - Cenário 2 sobre o balanceamento da linha de produção

Figura 11 - Representação do espaçamento de caminhões na linha de montagem

Figura 12 - Foto do interior de uma cabina de caminhão

Figura 13 - Contabilização da mudança de cor concurso ROADEF 2005

Figura 14 - Contabilização da mudança de cor no modelo matemático 